\title{
Mindfulness and mental toughness among provincial adolescent female hockey players
}

\author{
SP Walker, $\mathrm{PhD}$ \\ Unit for Professional Training and Services in the Behavioural Sciences \\ (UNIBS), Faculty of the Humanities, University of the Free State, \\ Bloemfontein, South Africa
}

\section{Corresponding author: SP Walker (walkersp@ufs.ac.za)}

Introduction: Mental toughness is highly valued within competitive sport. However, scant attention has been paid to the psychological processes that underpin mental toughness. Objectives: To explore the relationship between mindfulness and mental toughness among provincial adolescent female hockey players.

Methods: Provincial adolescent female hockey players $(\mathrm{N}=484)$ completed measures of mindfulness and mental toughness. Correlation coefficients were calculated with respect to mindfulness and mental toughness. A one-way between-groups analysis of variance (ANOVA) was conducted to determine whether athletes assigned to four levels of mindfulness (high, moderate, medium and low) differed significantly with regard to mental toughness.

Results: Mindfulness exhibited significant positive correlations with confidence, constancy and control, as well as with total mental toughness. The results of the ANOVA and the relevant post hoc analyses indicated that athletes in the high mindfulness group reported significantly higher levels of control and general mental toughness than those in the other three groups. The high mindfulness participants also reported significantly higher levels of constancy than those in the medium and low mindfulness groups. Conclusion: Mindfulness was positively correlated with all aspects of mental toughness investigated in this study. In addition, individuals with high levels of mindfulness reported higher control, constancy and general mental toughness than those with lower levels of mindfulness. Based on the current findings, the role of mindfulness in the development and maintenance of mental toughness among adolescent athletes warrants further investigation.

Keywords: mental toughness, mindfulness, athlete, adolescent, female

\section{S Afr J Sports Med 2016;28(2):46-50. DOI: 10.17159/2078-516X/2016/v28i2a1110}

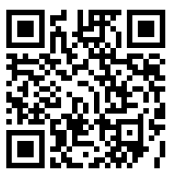

Mental toughness has come to be widely viewed as an important prerequisite for sustained athletic achievement. ${ }^{[1-2]}$ However, from a theoretical perspective, there is disagreement on the precise definition of mental toughness, while the effective and accurate operationalisation of mental toughness remains challenging at an empirical level.[3] Nevertheless, the general consensus appears to be that mentally tough athletes are resilient, goal-focused and confident in their abilities, as well as proficient at regulating their emotions and behaviour. [1-3] A body of research exploring various methods and techniques for enhancing mental toughness has emerged over the past decade. However, relatively little attention has been focussed on identifying the behavioural, cognitive and emotional mechanisms that bolster mental toughness. ${ }^{[4]}$ There is thus both a theoretical and practical need to explore the psychological basis of mental toughness from within existing, evidence-based psychotherapeutic, counselling and coaching paradigms.

Relatively few studies have evaluated the efficacy of psychological interventions aimed specifically at the development of mental toughness among athletes. The existing studies have focussed primarily on cognitivebehavioural interventions, which have generally demonstrated post-intervention improvements in mental toughness. ${ }^{[5-6]}$ However, cognitive-behavioural interventions aimed specifically at developing mental toughness do not appear to be superior to general mental skills development interventions when it comes to developing or increasing mental toughness. ${ }^{[6]}$ Recently, questions have been raised regarding evidence for the efficacy of mental skills interventions aimed at improving athletic performance and thus by implication, the efficacy of interventions purporting to develop or improve mental toughness. ${ }^{[7]}$ Based on the robust outcome data on mindfulness-based interventions in the field of clinical psychology, it has been suggested that a greater focus on mindfulness and mindfulness-based intervention might be indicated within the areas of sport and performance psychology in general, and with regard to mental toughness specifically. ${ }^{[7-8]}$

Mindfulness may be defined as the non-judgemental awareness of internal and external experiences as they occur in the present moment. ${ }^{[9]}$ Mindfulness-based approaches to performance enhancement advocate viewing emotions and cognitions as internal events, rather than objective or factual manifestations of reality that need to be changed or controlled to facilitate peak performance. ${ }^{[8 ; 10]}$ The core assumption of mindfulness-based approaches to performance enhancement is that once an individual is free of the need to control his or her thoughts and emotions they are in a position to optimally focus attentional resources outward on the execution of skills. This conceptualisation differs from that advocated by cognitive-behavioural interventions and traditional mental skills training, which promote the monitoring, evaluation and restructuring of cognition so as to enhance athletic performance by optimising the regulation of emotion and behaviour. [7]

To date, no studies have been published on the specific role of mindfulness in mental toughness. However, a number of researchers have investigated the influence of mindfulness on the wellbeing of athletes and, more generally, athletic 
performance. Mindfulness-based interventions have been shown to be associated with improved sporting achievement, increased self-confidence and the facilitation of performancepromoting states, such as flow. ${ }^{8,10]}$ The available evidence would seem to suggest that mindfulness is positively associated with a number of desirable sporting outcomes. However, the role of mindfulness in mental toughness remains unexamined. The aim of the current study was thus to explore the potential association of mindfulness with mental toughness among provincial adolescent female hockey players.

\section{Methods}

\section{Participants}

Ethical clearance to conduct the study was obtained from the relevant institutional body. The South African Hockey Association granted permission for data to be collected at annual female under-16 and under-19 interprovincial tournaments. Informed consent was obtained from all participants, as well as from the guardians of all minors, prior to the administration of the questionnaires. Data was gathered between matches or in the evenings under the supervision of the researcher or research assistant.

A total of 484 adolescent female hockey players participated in the study. The mean age of the sample was 16.2 years $(\mathrm{SD} \pm$ 1.2). Participants reported playing hockey competitively for an average of 8.4 years $(S D \pm 2.5)$. On average, participants had competed in 2.4 interprovincial tournaments $(\mathrm{SD} \pm 1.4$ ), with $6 \%$ having previously been selected for national teams.

\section{Measures}

The Sport Mental Toughness Questionnaire (SMTQ) was used to measure mental toughness. ${ }^{[11]}$ The SMTQ is a 14 item self-report questionnaire that yields a total mental toughness score, as well as scores on three subscales: confidence; constancy; and control. Responses to each item are indicated along a four-point Likert-type scale anchored by "not at all true" and "very true". Higher scores are indicative of higher levels of mental toughness. Acceptable construct validity and internal consistency have been reported for the SMTQ. [11]

The Child and Adolescent Mindfulness Measure (CAMM) was utilised as a measure of mindfulness. ${ }^{[12]}$ The CAMM is a ten-item self-report inventory with response options ranging from 'never true' to 'always true' along a five-point Likerttype scale. The CAMM yields a unitary mindfulness score, with higher scores indicating higher levels of mindfulness. The CAMM has demonstrated acceptable psychometric properties. [12]

The SMTQ and CAMM were translated into Afrikaans. Participants thus had the option of completing the questionnaires in either Afrikaans or English.

\section{Analysis}

Pearson's correlation coefficients were calculated for the CAMM total score, SMTQ total score, SMTQ confidence, SMTQ constancy and SMTQ control. Based on the distribution of the CAMM scores in the sample, participants were divided into four levels of mindfulness (low, medium, moderate and high). A one-way between-groups analysis of variance (ANOVA) was conducted to determine whether individuals in the four levels of mindfulness differed significantly with regard to their SMTQ subscale and total mean scores. Scheffé's post hoc test was used to determine the nature and direction of the significant differences determined by the ANOVA.

\section{Results}

The correlations between the SMTQ and the CAMM are presented in Table 1. Mean scores, SDs and internal consistency coefficients for each scale are also reported. It is apparent from Table 1 that all measures, with the exception of SMTQ Constancy scale $(\alpha=0.55)$, demonstrated acceptable levels of internal consistency and may thus be included in further analyses. ${ }^{[13]}$ Given that the SMTQ Constancy scale represents a theoretically valid MT construct, it was decided to include this scale in further analyses despite its poor internal consistency. The correlation coefficients in Table 1 suggest that mindfulness is positively and significantly correlated with all three SMTQ scales (confidence (Cnf): $p \leq 0.05$; constancy (Cst): $p \leq 0.01$; control (Ctl): $p \leq 0.01$ ), as well as with the SMTQ total score $(p \leq 0.01)$.

Given that there was a significant positive relationship between mindfulness and mental toughness, it was decided to investigate whether individuals reporting different levels of mindfulness differ significantly with regard to their level of mental toughness. Consequently, four levels (high, moderate, medium and low) of the independent variable (mindfulness) were created by dividing the sample into quarters based on the distribution of their CAMM scores (low: CAMM $\leq 18$; $n=129$; medium: CAMM 19 - 22; $n=118$; moderate: CAMM $23-26 ; n=121$; high CAMM $\geq 27 ; n=118$ ).

A one-way between-groups analysis of variance (ANOVA) was conducted to investigate differences between the four levels of the independent variable (mindfulness) with regard to mental toughness. Four dependent variables were included in the analysis: total mental toughness (SMTQ Tot); control (SMTQ Cnt); constancy (SMTQ Cst) and confidence (SMTQ Cnf). Preliminary assumption testing was conducted to check for normality, linearity, univariate and multivariate outliers, homogeneity of variance-covariance matrices and multicollinearity. No violations were detected. A statistically significant difference was apparent between the levels of mindfulness on the combined dependent variables $\left(F(9 ; 1168)=9.424 ; \quad p=0.0001 ;\right.$ Wilks ${ }^{\prime}=0.843$; partial $\left.\eta^{2}=0.055\right)$. Follow-up univariate ANOVAs were conducted to ascertain the specific SMTQ scales with regard to which the four mindfulness groups differed (Table 2).

Table 2 indicates that significant differences were apparent for level of mindfulness with regard to constancy (SMTQ Cst; $p=0.0001$ ), control (SMTQ Ctl; $p=0.0001$ ) and total mental toughness (SMTQ Tot; $p=0.0001$ ). The levels of constancy, control and total MT reported by the individuals in the four mindfulness groups thus differed to a statistically significant degree. However, when the relevant $f$-values are calculated (constancy: $f=0.05$; control: $f=0.15$; total mental toughness: $f=0.1$ ) it is apparent that while statistically significant, all three of these results reflect small to medium effect sizes and are thus of limited practical importance. ${ }^{[14]}$ 
Table 1. Pearson correlation coefficients, means, SDs and internal consistencies for SMTQ and CAMM (n = 484)

\begin{tabular}{|c|c|c|c|c|c|c|c|}
\hline & SMTQ Cnf & SMTQ Cst & SMTQ Ctl & SMTQ & CAMM & Mean $( \pm S D)$ & $\alpha$ \\
\hline CAMM & $0.12^{+}$ & $0.23^{*}$ & $0.39^{*}$ & $0.31^{*}$ & \multirow[t]{2}{*}{ _- } & $22.2( \pm 6.1)$ & 0.74 \\
\hline SMTQ & $0.79^{*}$ & $0.70^{*}$ & $0.71^{*}$ & _ & & $41.9( \pm 5.4)$ & 0.76 \\
\hline Tot & & & & & & & \\
\hline SMTQ Ctl & $0.25^{*}$ & $0.35^{*}$ & _ & & & $10.8( \pm 2.4)$ & 0.66 \\
\hline SMTQ Cst & $0.36^{*}$ & _ & & & & $13.5( \pm 1.9)$ & 0.55 \\
\hline SMTQ Cnf & & & & & & $17.6( \pm 3.0)$ & 0.72 \\
\hline
\end{tabular}

\section{${ }^{*} p \leq 0.01 ;{ }^{+} p \leq 0.05$}

CAMM = Child and Adolescent Mindfulness Measure; SMTQ Tot $=$ Sport Mental Toughness Questionnaire Total Score; SMTQ Cnf $=$ Sport Mental Toughness Questionnaire Confidence Scale; SMTQ Cst = Sport Mental Toughness Questionnaire Constancy Scale; SMTQ Ctl = Sport Mental Toughness Questionnaire Control Scale; $\alpha=$ Cronbach alpha coefficients

Table 2. Means, SDs and $F$-values for the one-way analysis of variance (ANOVA) for the four levels of mindfulness

\begin{tabular}{|c|c|c|c|c|c|c|}
\hline \multirow[t]{2}{*}{ SMTQ Scale } & High CAMM & $\begin{array}{l}\text { Moderate } \\
\text { CAMM }\end{array}$ & $\begin{array}{c}\text { Medium } \\
\text { CAMM }\end{array}$ & $\begin{array}{c}\text { Low } \\
\text { CAMM } \\
\end{array}$ & \multirow[t]{2}{*}{$F$-value } & \multirow[t]{2}{*}{$p$-value } \\
\hline & Mean $( \pm$ SD) & mean $( \pm S D)$ & mean $( \pm S D)$ & mean $( \pm S D)$ & & \\
\hline Confidence & $18.2( \pm 3.0)$ & $17.4( \pm 3.1)$ & $17.4( \pm 2.8)$ & $17.3( \pm 3.2)$ & 2.392 & 0.068 \\
\hline Constancy & $14.2( \pm 1.6)$ & $13.5( \pm 1.9)$ & $13.4( \pm 1.9)$ & $13.0( \pm 1.9)$ & $8.255^{*}$ & 0.0001 \\
\hline Control & $12.1( \pm 2.0)$ & $11.1( \pm 2.2)$ & $10.5( \pm 2.4)$ & $9.6( \pm 2.2)$ & $27.296^{*}$ & 0.0001 \\
\hline Total & $44.5( \pm 4.7)$ & $42.0( \pm 5.4)$ & $41.3( \pm 5.3)$ & $40.0( \pm 5.3)$ & $16.768^{*}$ & 0.0001 \\
\hline
\end{tabular}

$\mathrm{SMTQ}=$ Sport Mental Toughness Questionnaire; CAMM = Child and Adolescent Mindfulness Measure

Post hoc comparisons using the Scheffé test indicated that the high mindfulness group reported a significantly higher mean SMTQ total score $(44.5 ; \mathrm{SD} \pm 4.7)$ than the participants in the moderate $(42.0 ; \mathrm{SD} \pm 5.4 ; p=0.003)$, medium (41.3; $\mathrm{SD} \pm 5.3 ; p=0.000)$ and low $(40.0 \mathrm{SD} \pm 5.3 ; p=0.000)$ mindfulness groups. In addition, the high mindfulness group reported a significantly higher SMTQ control score $(12.1 ; \mathrm{SD} \pm 2.0)$ than the participants in the moderate $(11.1 ; \mathrm{SD} \pm 2.2 ; p=0.003)$, medium (10.5; $\mathrm{SD} \pm 2.4 ; p=0.000)$ and low $(9.6 \mathrm{SD} \pm 2.2 ; p=0.000)$ mindfulness groups. However, the mean SMTQ control score $(9.6, \mathrm{SD} \pm 2.2)$ reported by the low mindfulness group was only significantly lower than that reported by the moderate (11.1; $\mathrm{SD} \pm 2.2 ; p=0.000)$ and high (12.1; $\mathrm{SD} \pm 2.0$; $p=0.000$ ) mindfulness groups. Finally, the individuals in the high mindfulness group also reported higher mean SMTQ constancy scores (14.2; $\mathrm{SD} \pm 1.6)$ than those in the medium (13.4; $\mathrm{SD} \pm 1.9 ; p=0.009)$ and low (13.0; $\mathrm{SD} \pm 1.9 ; p=0.000)$ mindfulness groups.

\section{Discussion}

The results of the current study indicate that mindfulness is significantly and positively correlated with not only global mental toughness, but also with confidence, constancy and control. This suggests that as mindfulness increases among provincial female hockey players, so does their general level of mental toughness. In addition, increased mental toughness appears to be accompanied by more confidence in the player's ability to overcome challenges, improved adherence to a training regimen and more efficient self-regulation of competition-related behaviours and emotions. ${ }^{[1-2]}$ These findings are consistent with the existing literature with regard to mindfulness being positively associated with various cognitive processes and affective states necessary for success in athletic competition. ${ }^{[7-8,10]}$ Mindfulness may thus enable hockey players to respond more effectively to the behavioural demands of their competitive (e.g. tactical awareness and skills execution) and training (e.g. effortful training and skills acquisition) environments. More specifically, mindful athletes may be less inclined to becoming embroiled in unproductive attempts at cognitive and behavioural self-regulation. ${ }^{[9-10]}$ There appears to be value in implementing mindfulness interventions that have been shown to be effective in other sports within provincial adolescent hockey. ${ }^{[8,10,15]}$

The analyses of variance indicate that different levels of mindfulness are associated with differing levels of mental toughness across provincial female adolescent hockey players. More specifically, those participants classified as having high levels of mindfulness reported greater global mental toughness, as well as superior control compared to participants classified within the moderate, medium and low mindfulness categories. This suggests that adolescent female hockey players with high levels of mindfulness are significantly more capable of effectively dealing with the training and competition demands at the provincial level than players with lower levels of mindfulness. Furthermore, these players appear to be significantly more proficient at regulating their emotional and behavioural responses within the competitive environment than players with lower levels of mindfulness. It would thus seem that greater openness to experience, elevated levels of self-acceptance and superior efficiency at maintaining a present moment focus, facilitate a more effective approach to coping with challenges and dealing with competitive pressure. The current findings are in line with existing research highlighting mindfulness as an important component of sport-specific resilience. Particularly the ability to employ behavioural and emotional responses in service of one's sporting goals, rather than allocating these psychological resources in a manner that is inconsistent with one's performance objectives. ${ }^{[7-8]}$ The association between mindfulness and control among provincial adolescent female hockey players must, however, be interpreted more circumspectly than that between mindfulness and global mental toughness.

The current study indicates that the low mindfulness 
participants reported significantly lower global mental toughness only in comparison to the moderate and high mindfulness groups. Similarly, the hockey players in the high mindfulness group reported significantly higher levels of constancy only with respect to participants categorised as having low or medium levels of mindfulness. These findings suggest that the association between mindfulness and certain aspects of mindfulness (constancy and control) are more pronounced, and thus more relevant, at the distributional extremes of the current sample. This is, in all probability, as a result of the statistically pragmatic yet normatively arbitrary manner in which the sample was divided into four levels of mindfulness. It also underlines the practical value of viewing levels of mindfulness in athlete populations as continuous and fluid, rather than discrete. Furthermore, the small to medium effect sizes reported for the follow-up univariate ANOVAs indicate that the differences between the various levels of mindfulness are of limited practical value. Consequently, the differences in mental toughness between the mindfulness groups should be viewed as indicative of statistical trends rather than definitive real-world differences in mental toughness. ${ }^{[14]}$

There were no significant differences between the four mindfulness groups with regard to confidence. The literature does not offer an obvious explanation for this finding. However, it could be hypothesised that within this age cohort player confidence is still largely dependent upon external feedback regarding skill and competitive ability.[16] Consequently, as all the participants were provincial hockey players, it could be reasoned that little statistically significant variability should be expected with regard to their estimations of their abilities to successfully deal with the competitive challenges they face. A more theoretically consistent explanation might be that mindful athletes incline more toward a self-referenced basis for confidence, while less mindful athletes place greater emphasis on comparing their abilities to the situations they may face or to their peers. ${ }^{[7]}$ If this is the case, traditional measures of mental toughness and confidence may be inconsistent with a more mindfully-based conceptualisation of sport-specific confidence. A more pragmatic view might be that confidence is a prized and much emphasised attribute at all levels of performance. As such, measures of confidence could be vulnerable to positive response bias, resulting in the distribution of scores within the sample being excessively skewed in the direction of high sporting confidence.

\section{Study Limitations}

The study is not without limitations. First, a cross-sectional correlational design was used, thus no conclusions can be drawn regarding possible causal relationships between mindfulness and mental toughness. Future studies might use more sophisticated modelling methods to investigate possible pathways of causality between mindfulness and mental toughness. Intervention studies could then be embarked upon to determine the effect of mindfulness-based sport performance interventions on mental toughness. Second, a very homogenous sample was used in this study with obvious implications regarding the generalisability of the findings beyond the current sample. Consideration should be given to replicating the study in individual sports and among male athletes, as well as in mixed gender samples. Third, the current study only explored the relationship between mindfulness and mental toughness. It is also necessary to determine the relationship between mental toughness and other constructs within the increasing number of mindfulness-oriented performance interventions, such as acceptance, cognitive defusion and values clarification. ${ }^{\text {7] }}$ Finally, the CAMM is a generic measure of mindfulness. Consequently, it might be useful to adapt the CAMM or otherwise develop a sport-specific measure of mindfulness for use in studies of this nature.

\section{Conclusion}

While exploratory in nature, the current study provides evidence of a positive association between mindfulness and mental toughness among provincial adolescent female hockey players. It appears that players reporting higher levels of mindfulness demonstrate greater global mental toughness, are more consistent in their approach to their sport and are more proficient in regulating their behaviour and emotions within the competitive environment. Notwithstanding the limitations of the study and the need for further research, the promotion of mindfulness could be expected to have a positive effect on the mental toughness of this specific sporting population.

Acknowledgements: The author would like to thank Mrs. Hanli van der Merwe for her assistance with data collection and Prof Karel Esterhuyse for his comments on an earlier draft of this paper.

\section{References}

1. Jones G, Hanton, S, Connaughton D. A framework of mental toughness in the world's best performers. Sport Psychol 2007;21(2):243-264. doi:10.1123/tsp.21.2.243.

2. Sheard M. Mental toughness: The mindset behind sporting achievement. New York. Routledge, 2010.

3. Gucciardi DF, Hanton S, Gordon S, et al. The concept of mental toughness: tests of dimensionality, nomological network, and $\begin{array}{llll}\text { traitness. J } & \text { J }\end{array}$ [http://dx.doi.org/10.1111/jopy.12079]

4. Connaughton D, Thelwell R, Hanton S. Mental toughness development: issues, practical implications and future direction. In Gucciardi DF, Gordon S. Mental toughness in sport: developments in theory and research. New York: Routledge, 2011.

5. Parkes JF, Mallet CJ. Developing mental toughness: Attributional style retraining in rugby. Sport Psychol 2011; 25(3):269-287. doi: 10.1122/tsp.25.3.269

6. Gucciardi DF, Gordon S, Dimmock JA. Evaluation of a mental toughness training program for youth-aged Australian footballers: I A quantitative analysis. J Appl Sport Psychol 2009; 21(3):307-323. [http://dx.doi.org/10.1080/1041320093026066]

7. Gardner FL, Moore ZE. The psychology of enhancing human performance: the Mindfulness-Acceptance-Commitment (MAC) approach. New York: Springer, 2007.

8. Sappington R, Longshore K. Systematically reviewing the efficacy of mindfulness-based interventions for enhanced athletic performance. J Clin Sport Psychol 2015;9(3):232-262. [http://dx.doi.org/10.1123/jcsp.2014-0017]

9. Baer RA. Mindfulness training as a clinical intervention: A conceptual and empirical review. Clin Psychol Sci Prac 2003;10(2):125-143. [http://dx.doi.org/10.1093/clipsy. bpg016] 
10. Pineau TR, Glass C, Kaufman KA, et al. Self- and team-efficacy beliefs of rowers and their relation to mindfulness and flow. J Clin Sport Psychol 2014;8(2):142[http://dx.doi.org/10.1123/jcsp.2014-0019]

11. Sheard M, Golby J, van Wersch A. Progress toward construct validation of the Sport Mental Toughness Questionnaire (SMTQ).

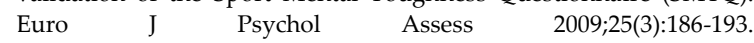
[http://dx.doi.org/10.1027/1015-5759.25.3.186]

12. Greco LA, Baer RA, Smith GT. Assessing mindfulness in children and adolescents: Development and validation of the Child and Adolescent Mindfulness Measure (CAMM). Psychol Assess 2011;23(3):606-614. [http://dx.doi.org/10.1037/a0022819]
13. Foster JJ, Parker I. Carrying out investigations in psychology: methods and statistics. Leicester: The British Psychological Society, 1999.

14. Cohen J. Statistical power analysis for the behavioral sciences. 2nd ed. New York. Academic Press, 1988.

15. Thompson RW, Kaufman KA, De Petrillo LA, et al. One year followup of Mindful Sport Performance Enhancement (MSPE) with archers, golfers and runners. J Clin Sport Psychol 2011;5(2):99-116. doi: 10.1123/jesp.5.2.99

16. Magyar TM, Feltz DL. The influence of dispositional and situational tendencies on adolescent girls' sport confidence sources. Psychol Sport Exerc 2003:4(2):175-190. [http://dx.doi.org/10.1016/S14690292(01)00037-1] 